\title{
The immunomodulatory effect of green tea (Camellia sinensis) leaves extract on immunocompromised Wistar rats infected by Candida albicans
}

\author{
Retno P. Rahayu ${ }^{1}$, Remita A. Prasetyo², Djoko A. Purwanto ${ }^{3}$, Utari Kresnoadi ${ }^{4}$, Regina P. D. Iskandar ${ }^{5}$ and \\ Muhammad Rubianto ${ }^{6}$ \\ 1. Department of Oral and Maxillofacial Pathology, Faculty of Dental Medicine, Universitas Airlangga, Surabaya, \\ Indonesia; 2. Installation of Oral and Dental Health, Dr. Soetomo Hospital, Surabaya, Indonesia; 3. Department \\ of Pharmaceutical Chemistry, Faculty of Pharmacy, Universitas Airlangga, Surabaya, Indonesia; 4. Department of \\ Prosthodontics, Faculty of Dental Medicine, Universitas Airlangga, Surabaya, Indonesia; 5. Student of Immunology, \\ Postgraduate School, Universitas Airlangga, Surabaya, Indonesia; 6.Department of Periodontics, Faculty of Dental \\ Medicine, Universitas Airlangga, Surabaya, Indonesia. \\ Corresponding author: Retno P. Rahayu, e-mail: a-retno-p-r@fkg.unair.ac.id \\ Co-authors: RAP: mitaprasetyo@gmail.com, DAP: djokoagus@ff.unair.ac.id, UK: utari-k@fkg.unair.ac.id, \\ MR: rubianto@fkg.unair.ac.id, RPDI: regina_pdewi@hotmail.com \\ Received: 03-01-2018, Accepted: 02-05-2018, Published online: 07-06-2018
}

doi: 10.14202/vetworld.2018.765-770 How to cite this article: Rahayu RP, Prasetyo RA, Purwanto DA, Kresnoadi U, Iskandar RPD, Rubianto M (2018) The immunomodulatory effect of green tea (Camellia sinensis) leaves extract on immunocompromised Wistar rats infected by Candida albicans, Veterinary World, 11(6): 765-770.

\begin{abstract}
Background and Aim: The immunocompromised condition is considered a defect in the immune system. This condition tends to increase the risk of oral candidiasis, due to the inability of the immune system to eliminate the adhesion of Candida albicans and leads to systemic candidiasis with a mortality rate of $60 \%$. Green tea (Camellia sinensis) contains potential antioxidant and immunomodulatory which acts as anticancer, antifungal, and antivirus agent. The aim of this study was to invent herbal-based medicine, which acts as an immunomodulator and antifungal agent to treat fungal infection in immunocompromised patients.
\end{abstract}

Materials and Methods: Thirty-five immunocompromised Wistar rats induced with C. albicans were divided into 7 groups $(\mathrm{n}=5)$ : Control group $(\mathrm{C}+)$; treated for 4 days with green tea extract $1.25 \%(\mathrm{GT} 4)$, epigallocatechin gallate (EGCG) $1 \%$ (EGCG 4), EGC 1\% (EGC 4); and treated for 7 days with green tea extract 1.25\% (GT 7), EGCG 1\% (EGCG 7), and EGC $1 \%$ (EGC 7). Tongue tissue was collected and analyzed with immunohistochemistry staining using monoclonal antibody; interleukin (IL)-17A, IL-8, and human beta-defensin 2 (HBD)-2. Data were analyzed using analysis of variance test and Tukey honest significant differences test.

Results: The expression of IL-17A, IL-8, and HBD-2 was significantly increased $(\mathrm{p}=0.000)$ after green tea extract administration in 7 days, whereas in 7 days, the expression of IL-8, IL-17A, and HBD-2 after EGCG and EGC administration did not give a significant result ( $\mathrm{p}>0.005)$.

Conclusion: Within the limits of this study, green tea extract has the ability as an immunomodulatory agent in an immunocompromised patient infected by $C$. albicans through expression augmentation of IL-8, IL-17A, and HBD-2 compared to EGCG and EGC.

Keywords: epigallocatechin gallate, epigallocatechin, green tea extract, immunocompromised, oral candidiasis.

\section{Introduction}

The immune system is an essential mechanism to against microorganism and its toxins. The defect in the immune system could lead to an immunocompromised condition that put patients at high risk of various infections of fungal, viral, and bacterial infections [1]. One of the most common infection is fungal infection, oral candidiasis, which could lead to systemic candidiasis with a mortality rate of $60 \%[1,2]$.

Copyright: Rahayu, et al. Open Access. This article is distributed under the terms of the Creative Commons Attribution 4.0 International License (http://creativecommons.org/licenses/ by/4.0/), which permits unrestricted use, distribution, and reproduction in any medium, provided you give appropriate credit to the original author(s) and the source, provide a link to the Creative Commons license, and indicate if changes were made. The Creative Commons Public Domain Dedication waiver (http:// creativecommons.org/publicdomain/zero/1.0/) applies to the data made available in this article, unless otherwise stated.
Candida albicans is part of the indigenous microbial flora in humans and can be found in the oral cavity, and is unique among opportunistic pathogens because it is part of the normal microbial flora of the host $[1,3]$. C. albicans has been shown to play an important role in oral candidiasis, denture stomatitis, and severe periodontitis. In immunocompromised patients, mucosal immunity is not able to eliminate the adhesion of C. albicans. The prevalence of oral candidiasis tends to increase along with immunocompromised patients, such as human immunodeficiency virus (HIV), diabetes mellitus, iatrogenic infections, and cancer [4]. The condition causes impaired phagocytosis effect of polymorphonuclear cells, and macrophages decrease the quality and quantity of cytokines [5]. C. albicans infection triggers differentiation of $\mathrm{T}$ helper (Th) cell into Th17 to produce Interleukin 17 (IL-17) through activation IL-23 that was produced by dendritic cells. 
IL-17A plays an important role in mobilization and fungicidal activity of neutrophil $[5,6]$. In addition, activation of IL-17A through receptor IL-17R in epithelial induce the release of human beta-defensin 2 (HBD-2). HBD-2 is part of innate immunity system that plays a role in recruitment and activation of neutrophil to phagocyte against C. albicans [7].

The resistance to antifungal agents tends to increase nowadays and causes failure of treatment. Therefore, innovation is needed to enhance the effectivity of antifungal agents. The common anti-fungal agents gradually become more unaffordable for some Indonesians. Therefore, it is required to seek a solution to treat oral candidiasis in immunocompromised patients properly.

Current researches which are supported by Ministry of Technology and Higher Education tend to use more natural ingredients. Herbal based medicines could be the proper solution to treat some diseases. In addition, herbal medicines are abundantly provided in nature, affordable, have a low toxicity level, and are recognized safe by Food and Drug Administration (FDA) [8]. Catechin that contained in green tea could have higher bioavailability when absorbed orally.

One of the natural ingredients that are widely consumed by Indonesians is Camellia sinensis (green tea), which is known to act as a potent antioxidant. It contains catechins that consist of epicatechin (EC), epigallocatechin (EGC), EC gallate, and EGC gallate (EGCG). It is reported that catechin in green tea has the ability as antioxidant, anticancer, antifungal, and antivirus [9]. EGCG and EGC act as an immunomodulator by influencing the proliferation of lymphocyte $\mathrm{T}$ and cytokines production. Green tea extract enhances lymphoblast to induce the production of lymphocytes, while EGCG stimulates the production of IL- $1 \alpha$, IL-1 $\beta$, monocytes, and lymphocytes [10].

The EGCG and EGC administered orally could suppress inflammation, inhibit proliferation and pro-inflammation cytokines, as well as, inhibit the activation of NF-KB [11]. This condition leads to lower level of IL-8 which plays as chemoattractant and neutrophil recruitment. The active component is responsible for biological effects such as immunomodulator, anti-tumor activity, and antimicrobial [12].

It is possible that green tea possesses immunomodulatory effect against oral candidiasis in immunocompromised patients. However, further investigation is needed to study the immunomodulatory effect of green tea extract, EGCG, EGC through increasing expression of IL-8, IL-17A, and HBD-2. This aim of this study was to develop natural based immunomodulator and anti-fungal agent to treat fungal infection in immunocompromised patients.

\section{Materials and Methods}

\section{Ethical approval}

This study has been approved by the Commission of Ethical Clearance, Faculty of Dental Medicine,
Universitas Airlangga, Surabaya, Indonesia (No. 306/ HRECC.FODM/XXI/2017).

\section{Animals}

The research was post-test only control group design using Wistar rats as an animal model. The criteria of rats were healthy, 3 months old, and weighing around $250 \mathrm{~g}$. This study using 35 immunocompromised Wistar rats induced with $C$. albicans, which are divided into 7 groups $(\mathrm{n}=5)$ : Control group $(\mathrm{C}+)$; treated with green tea extract concentration $1.25 \%$ for 4 (GT 4) and 7 (GT 7) days; EGCG 1\% for 4 (EGCG 4) and 7 (EGCG 7) days; and treated EGC 1\% for 4 (EGC 4) and 7 (EGC 7) days.

\section{Experimental design}

This study was conducted in Integrated Research and Testing Laboratory Biochemistry Faculty of Medicine, Universitas Airlangga. Immunocompromised condition was induced by Dexamethasone $0.8 \mathrm{mg} / \mathrm{kg}$ and Tetracycline $12 \mathrm{mg} / \mathrm{kg}$ intraperitoneally for 7 days. Blood serums were collected on day 1 and 7 to measure the immunocompromised condition through a blood test. The immunocompromised rats were then induced with C. albicans, by inoculating $0.1 \mathrm{~mL}$ saline suspension which contains $3 \times 10^{8} \mathrm{C}$. albicans to tongue tissue using cotton rolls in all groups for 6 times in day 7-12. Treatment groups were treated with green tea extract, EGCG and EGC until 16 and 19 days, respectively, based on its group treatment.

\section{Preparation of green tea extract}

Two and half gram of dried green tea leaf was mixed into $200 \mathrm{~mL}$ of boiling distilled water for $30 \mathrm{~min}$. After being rested and filtered, the extract was freeze-dried to preserve the component substances green tea extract with a concentration of $1.25 \%$ was made by mixed $12.5 \mathrm{mg}$ of dried green tea extract with $100 \mathrm{~mL}$ of distilled water for $30 \mathrm{~min}$. It was followed by filtering the extract.

\section{Preparation of EGCG and EGC extract}

The EGC and EGC powder was obtained from Xi'an Rongsheng Biotechnology Co., Ltd, Xi'an, PRC. There were $2.5 \mathrm{~g}$ of EGCG and EGC powder in $250 \mathrm{~mL}$ sterilized distilled water needed to make $1 \%$ EGCG and EGC solution. The solution was double filtered $30 \mathrm{~min}$ after mixture to gain clear EGCG and EGC solution.

\section{Immunohistochemistry assay (IHC)}

The rats were decapitated using a combination of Ketamine and Xylazine (100 mg/kg and $10 \mathrm{mg} \mathrm{kg} / \mathrm{BB})$ intramuscular injection. $\mathrm{C}+$ group was terminated on day 13; while GT 4, EGC 4, and EGCG 4 groups were terminated on day 16; and green tea 7, EGCG 7, and EGC 7 were terminated on day 19. Tongue tissues were collected and analyzed using IHC staining using monoclonal antibody of IL-17A, IL-8, and HBD-2 from Santa Cruz Biotech (Santa Cruz Biotechnology Inc., Dallas, Texas, USA) and examined under 


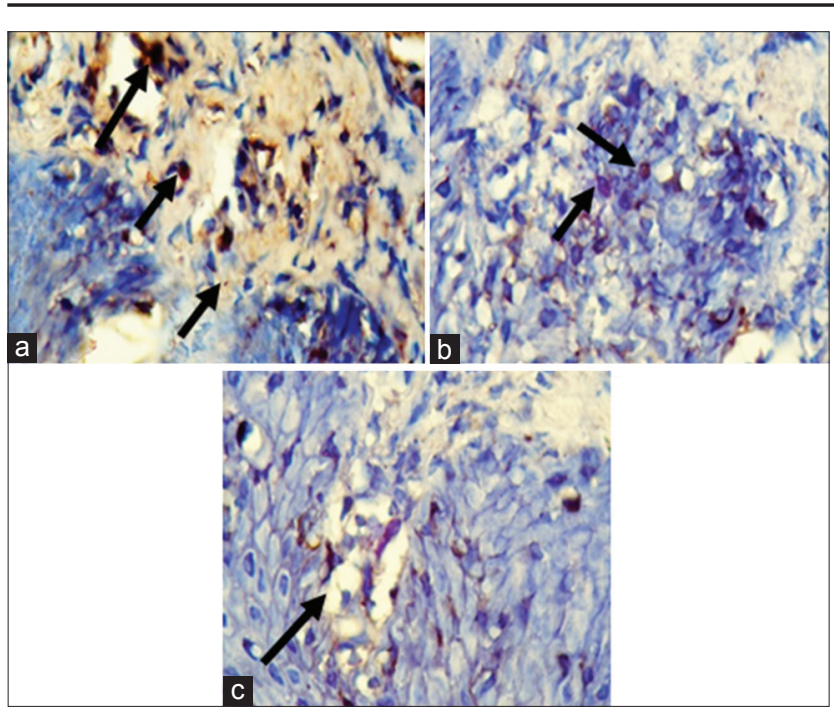

Figure-1: Immunohistochemistry of interleukin-8 expression (black arrow) in tongue tissue $(400 \times)$ in the group which treated with green tea extract (a), epigallocatechin (EGC) gallate (b) and EGC (c) on day 7.

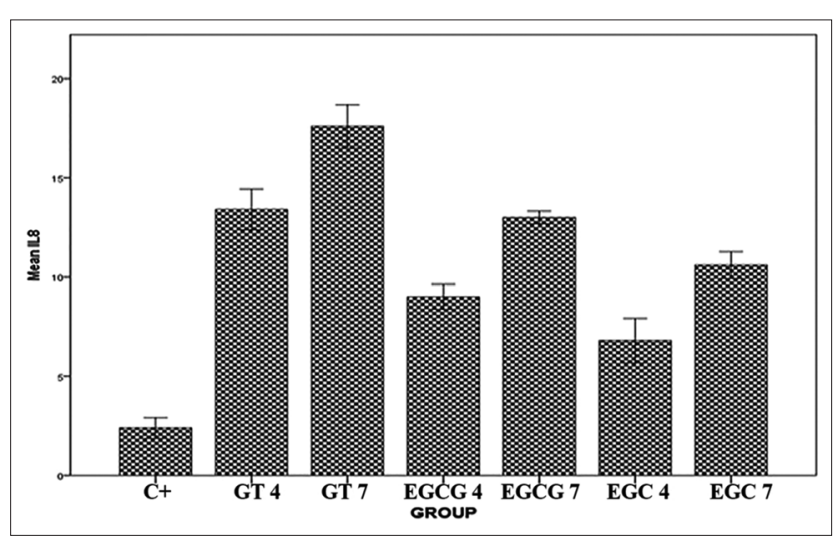

Figure-2: The mean of interleukin-8 expression level after treated with green tea extract, epigallocatechin (EGC) gallate, and EGC.

Olympus digital microscope (Olympus Co., Tokyo, Japan) at $400 \times$ magnification.

\section{Statistical analysis}

The data were analyzed using SPSS software version 10.05 (SPSS Inc., Chicago, USA) with ANOVA and post hoc Tukey honest significant differences (HSD) test.

\section{Results}

The IHC results of IL-8 expression are showed in Figure-1. Mean of each group for IL-8 expression was shown in Figure-2. ANOVA results showed that the expression of IL-8 was significantly different for each group ( $\mathrm{p}=0.000$ ). Tukey HSD test results in 4 days showed there was significant difference expression between GT 4 group compared to EGCG4 $(\mathrm{p}=0.011)$ and EGC4 $(\mathrm{p}=0.000)$ groups. However, there was no significant difference of IL-8 expression between EGCG 4 and EGC 4 groups ( $\mathrm{p}=0.495)$. The expression of IL-8 in day 7 was higher than in day 4 . Tukey HSD test result showed greater expression of IL-8 in
GT 7 group compared to EGCG $7(p=0.000)$ and EGC $7(p=0.000)$ groups. The expression of IL- 8 between EGCG 7 and EGC 7 groups did not show a significant difference $(\mathrm{p}=0.394)$.

The expression of IL-17A is shown in Figure-3, and the mean is shown in Figure-4. The expression of IL-17A was significantly different in each group $(p=0.000)$. Tukey HSD test results in 4 days showed there was a significant difference between GT 4 group compared to EGCG $4(p=0.010)$ and $\mathrm{EGC} 4(\mathrm{p}=0.000)$ groups. Meanwhile, the comparison of IL-17A expression between EGCG 4 and EGC 4 did not show a significant difference $(\mathrm{p}=0.076)$. The expression of IL-17A on day 7 is higher than on day 4. HSD test result showed higher expression of IL-17A in GT 7 group compared to EGCG $7(p=0.000)$ and EGC 7 $(p=0.000)$ groups. The expression of IL-17A between EGCG 7 and EGC 7 groups did not show a significant difference $(\mathrm{p}=0.110)$.

The expression of HBD-2 is shown in Figure-5, while the means are shown in Figure- 6 . The expression of HBD-2 was significantly different for each group ( $p=0.000$. Tukey HSD test result showed no significant difference in HBD-2 expression in GT 4 group compared to EGCG 4 group $(\mathrm{p}=0.080)$ but showed a significant difference if compared to EGC 4 group ( $\mathrm{p}=0.023)$. The expression of HBD-2 between EGCG 4 and EGC4 groups did not show any significant difference $(\mathrm{p}=0.998)$. The expressions of HBD-2 in day 7 are greater than on day 4. Tukey HSD test result showed that the expression of HBD-2 in GT 7 group was significantly different compared to EGCG $7(p=0.000)$ and EGC $7(p=0.001)$ groups. Meanwhile, the expression of HBD-2 between EGCG 7 and EGC 7 groups did not show a significant difference $(p=0.998)$.

\section{Discussion}

The current management of oral candidiasis is not effective enough, especially for immunocompromised individuals, due to high risk of recurrence. Therefore, adjunctive therapy is needed to get a better result. Recently, approximately $80 \%$ of antimicrobial, cardiovascular, immunosuppressive, and anticancer drugs are of plant origin. It is widely accepted that more than $80 \%$ of drug substances are either directly derived from natural products or developed from a natural compound [13]. One of the plants that have a therapeutic effect is green tea (Camellia Sinensis). Green tea extract is known to have anti-oxidant and antifungal properties [14].

Prevalence of oral candidiasis tends to increase each year as increasing number of the immunocompromised patient due to diabetes mellitus, prolonged consumption immunosuppressant and antibiotic, transplant organ recipient, cancer survivor, and patient with HIV/AIDS [15]. Prolonged and irrational usage of anti-fungal (azole) is believed to contribute to the higher prevalence of oral candidiasis, which leads 


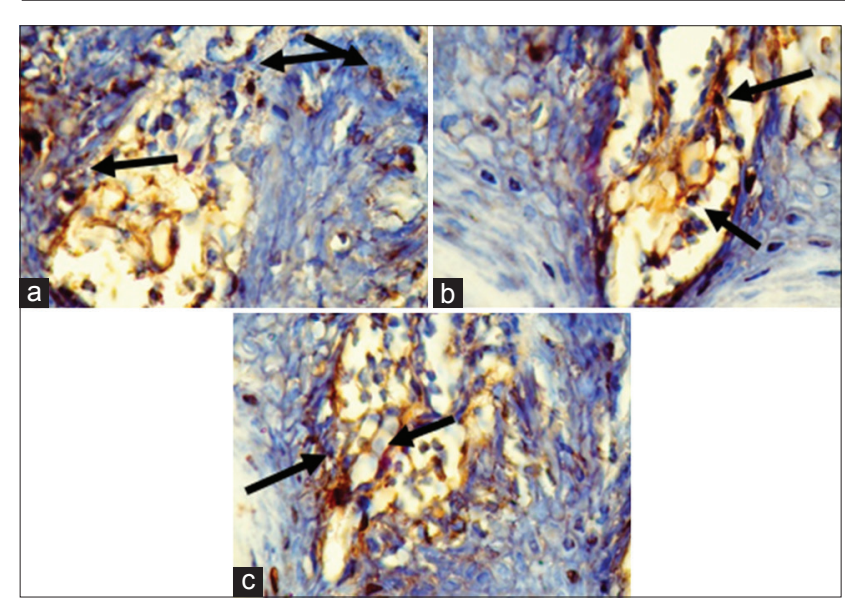

Figure-3: Immunohistochemistry of interleukin-17A expression (Black arrow) in tongue tissue $(400 x)$ of the group with green tea extract (a), epigallocatechin (EGC) gallate (b), EGC (c) on day 7.

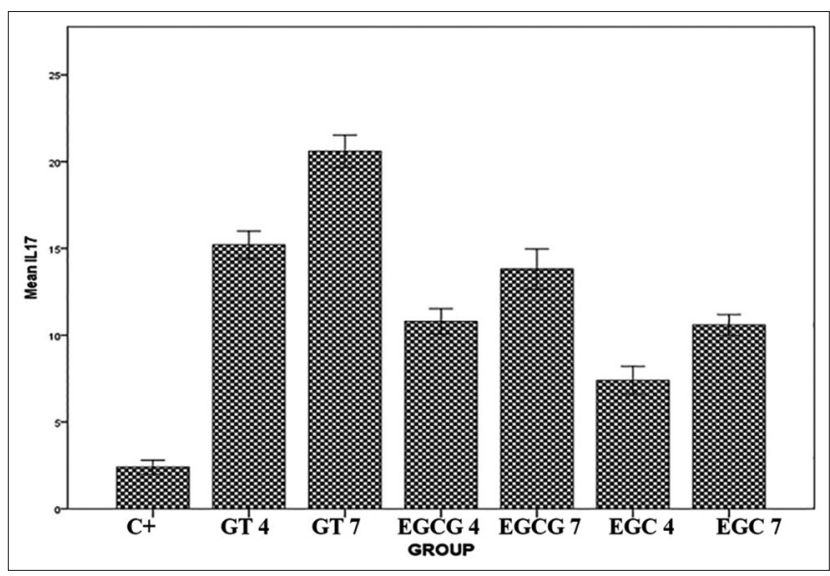

Figure-4: Interleukin-17A expression level after treated with green tea extract, epigallocatechin (EGC) gallate, and EGC.

to treatment failure [16]. Therefore, innovation to enhance anti-fungal effectivity is needed. In immunocompetent patients, every microorganism including fungi is controlled by the immune response, whether specific or non-specific. The immune system of immunocompromised patient fails to eliminate the adhesion of Candida that leads to oral candidiasis [6].

Several antifungal agents, nystatin, and azole are reported to be resistant to oral candidiasis. Meanwhile, improper treatment of oral candidiasis may lead to systemic infection of Candida that increases mortality rate to $60 \%$ [17]. Green tea extract which contains EGCG and EGC is considered as an herbal-based therapy for oral candidiasis. Green tea extract is generally recognized as safe by the FDA Safety of the USA [8], because of green tea that is traditionally consumed as food relies on that history of food use as evidence of safety.

This study showed that IL-8 is increased on day 4 and 7 after treated green tea extract. Green tea extract showed higher IL-8 expression compare to EGCG and EGC due to green tea extract contains various active components which synergistically increase IL-8 expression. IL-8 is important due to chemo-attractant properties which recruit neutrophils for phagocytosis to inhibit $C$. albicans colony formation. A previous study by Steubesand et al. [18] showed oral administration of EGCG and EGC could inhibit the synthesis of pro-inflammation cytokines and the activation of NF- $\mathrm{KB}$ which leads to decreasing IL-8 expression and increasing HBD-2. Various components contain in green tea extract work synergistically to enhance the expression of IL-17A and IL-8 which play as a chemoattractant to release HBD-2.

The expression of IL-17A is shown in Figure-4 after treated with green tea extract was significantly different in each group $(\mathrm{p}=0.000)$. Meanwhile, the comparison of IL-17A expression between the group treated with EGCG and EGC did not show a significant difference $(p=0.076)$ in day- 7 is higher than on day-4. There is the IL-17A play vital role in mobilization and activity of neutrophils against infection, and it will be expressed by the activity of Th17 through ROR $\gamma \mathrm{t}$ and increases the number of neutrophils to inhibit oral candidiasis, even in immunocompromised patients $[19,20]$. Green tea extract contains flavonoids which have an immunomodulatory effect, thus enhance IL-2 production which activates the proliferation and differentiation of $\mathrm{T}$ cells differentiate into Th1 and Th2, Th1 cells secrete various cytokines Interferon gamma (IFN- $\gamma$ ) to activate macrophages [21]. Flavonoid contained in green tea extract enhances proliferation of lymphocytes could affect CD4+ cells to activate Th17 and produces IL-17A [22]. It is followed by epithelial induction through IL-17R, which activates the synthesis of HBD-2, IL-8, and G-CSF. Deficiency of IL-17A led to a neutrophil deficiency which increases the risk of systemic $C$. albicans infection.

The non-specific immune response is triggered as $\alpha$-mannans binds to $C$. albicans cell wall. The binding stimulates specific immune response through Th17. The activity of Th17 cells through inducing Treg will reduce expression of Dectin-2 in C. albicans [23]. These conditions stimulate the expression of various markers such as ROR $\gamma \mathrm{t}$, IL-17A, and HBD2 , as well as the number of neutrophils. A sufficient number of $C$. albicans might be increased and initiate oral candidiasis.

Green tea extract, EGC, and EGCG inhibit Candida biofilm formation and preformed biofilm. Higher EGCG concentration is reported to be substantial for in vivo activity of C. albicans proteasome chymotrypsin-like. Proteasome activities disturb cellular metabolism and yeast structure [15]. In C. albicans infection, macrophages release various pro-inflammation cytokines supported by IL-23 such as IL-17A, IL-21, and IL-22, and would trigger differentiation of Th into Th1, Th2, and Th17. IL-17A is the most prominent cytokines released by Th17 that was activated by IL-23. It is beneficial to trigger epithelial to secrete antimicrobial peptides and recruit neutrophils for phagocytosis, C. albicans in particular [10,12]. In certain condition, there would be declined secretion of IL-17 due to such mechanism by C. albicans to avoid 


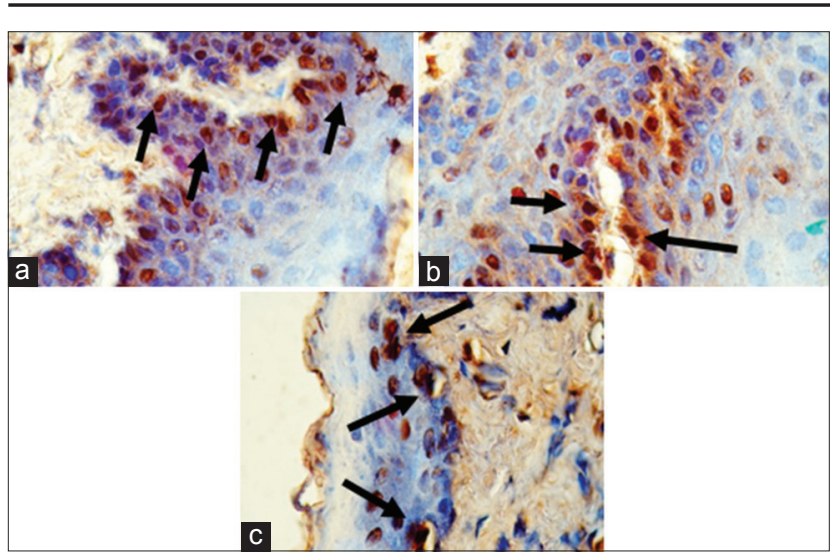

Figure-5: Immunohistochemistry of human betadefensin-2 expression (black arrow) (400x) of the group treated with green tea extract (a), epigallocatechin (EGC) gallate (b), EGC (c) on day 7.

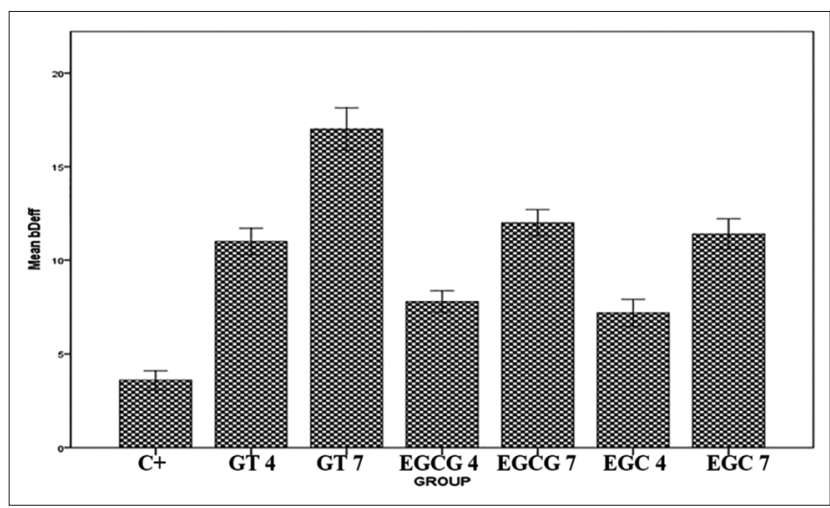

Figure-6: Human beta-defensin-2 expression level after treated with green tea extract, epigallocatechin (EGC) gallate, and EGC.

the host immune response.

HBD-2 expressions are higher in green tea extract group compared to EGC and EGCG. Higher expression HBD-2 in green tea extract group due to its ability to activate HBD-2 release as effector molecules in oral mucosal innate immunity. HBD-2 is part of mucosal primary defense mechanism against $C$. albicans through several mechanisms in the cell membrane, such as binding ions $\mathrm{Ca} 2+$ and $\mathrm{Mg} 2+$ [24]; consequently, there would be ion and peptide exchange that interfere the stability of $C$. albicans membrane. Instability of membrane leakage would lead to apoptosis of C. albicans. This shows that various components contained in green tea extract work synergistically and simultaneously to increase IL-8 and IL-17A expressions. Lack of HBD-2 leads to reduce recruitment and activation of neutrophils and affects its phagocytic ability against $C$. albicans. Miramón et al. [25] stated that oral candidiasis can be cured in immunocompromised individuals. Decreasing number of HBD-2 stimulates reduce the activity and recruitment of neutrophil moreover lower phagocytosis activity against $C$. albicans.

\section{Conclusion}

The expressions of IL-17A, IL-8, and HBD-2 are higher after treated with green tea extract, compared to treatment with EGC and EGCG. The results show that green tea extract gives better immunomodulatory effect, compared to EGC and EGCG. It is concluded that green tea extracts possess immunomodulatory effect against $C$. albicans infection in immunocompromised patients by increasing the expression of IL-8, IL-17A, and HBD-2.

\section{Authors' Contributions}

All authors participated equally in the study plan and design. RAP, UK, and RPDI collected the samples from the farms and prepared the animal models. RPR and DAP assisted in the laboratory work. MR carried out the statistical analysis of data and reporting the results. All authors collaborated in writing, revising, and improvement of the article for publication.

\section{Acknowledgments}

The present study received financial support from The Ministry of Research and Higher Education of The Republic of Indonesia under the PDUPT scheme with Grant No. 018/SP2H/LT/DRPM/II/2016.

\section{Competing Interests}

The authors declare that they have no competing interests.

\section{References}

1. Sganga, G. (2011) Fungal infections in immunocompromised patients. Mycoses, 54(4): 1-3.

2. Gow, N.A. and Hube, B. (2012) Importance of the Candida albicans cell wall during commensalism and infection. Curr. Opin. Microbiol., 15(4): 406-412.

3. Fidel, P.L. Jr. (2011) Candida-host interactions in HIV disease: Implications for oropharyngeal candidiasis. $A d v$. Dent. Res., 23(1): 45-49.

4. Williams, D. and Lewis, M. (2011) Pathogenesis and treatment of oral candidosis. J. Oral Microbiol., 3(57): 71-82.

5. Mayer, F.L., Duncan, W.D. and Hube, B. (2013) Candida albicans pathogenicity mechanisms. Virulence, 4(2): 119-128.

6. Smeekens, S.P., van de Veerdonk, F.L., Kullberg, B.J. and Netea, M.G. (2013) Genetic susceptibility to Candida infections. EMBO Mol. Med., 5(6): 1-9.

7. Hernández-Santos, N. and Gaffen, S.L. (2012) Th17 cells in immunity to Candida albicans. Cell Host Microbe, 11(5): 425-435.

8. Abdel-Rahman, A., Anyangwe, N., Carlacci, L., Casper, S., Danam, R.P., Enongene, E., Erives, G., Fabricant, D., Gudi, R., Hilmas, C.J. and Hines, F. (2011)The safety and regulation of natural products used as foods and food ingredients. Toxicol. Sci., 123(2): 333-348.

9. Tanaka, T., Matsuo, Y. and Kouno, I. (2013) Biochemical and physicochemical characteristics of green tea polyphenols. In: Juneja, L.R., Kapoor, M.P., Okubo, T. and Rao, T.P., editors. Green Tea Polyphenols, Nutraceuticals of Modern Life. CRC Press, Boca Raton. p19-29.

10. Wu, D., Wang, J., Pae, M. and Meydani, S.N. (2012) Green tea EGCG, T cells, and T cell-mediated autoimmune diseases. Mol. Aspects Med., 33(1): 107-118.

11. Di Lorenzo, C., Dell'Agli, M., Sangiovanni, E., Dos Santos, A., Uberti, F., Moro, E., Bosisio, E. and Restani, P. (2013) Correlation between catechin content and NF-kB inhibition by infusions of green and black tea. Plant Foods Hum. Nutr., 68(2): 149-154.

12. Kumar, V. and Sharma, A. (2010) Neutrophils: 
Cinderella of innate immune system. Int. Immunopharmacol., 10: 1325-1334.

13. Pan, S.Y., Zhou, S.F., Gao, S.H., Yu, Z.L., Zhang, S.F., Tang, M.K., Sun, J.N., Ma, D.L., Han, Y.F., Fong, W.F. and Ko, K.M. (2013) New perspectives on how to discover drugs from herbal medicines: Cam's outstanding contribution to modern therapeutics. Evid. Based Complement. Alternat. Med., 2013: 627375.

14. Kim, H.S., Quon, M.J. and Kim, J.A. (2014) New insights into the mechanisms of polyphenols beyond antioxidant properties; lessons from the green tea polyphenol, epigallocatechin 3-gallate. Redox Biol.,2: 187-195.

15. Sardi, J.C., Scorzoni, L., Bernardi, T, Fusco-Almeida, A.M. and Mendes, G.M.J. (2013) Candida species: Current epidemiology, pathogenicity, biofilm formation, natural antifungal products and new therapeutic options. $J$. Med. Microbiol., 62: 10-24.

16. Murata, Y., Isobe, T., Kofuji, K., Nishida, N. and Kamaguchi, R. (2013) Development of film dosage forms containing miconazole for the treatment of oral candidiasis. Pharmacol. Pharm., 4: 325-330.

17. Gaona-Flores, V., Quiróz-Guzmán, R., CervantesTovar, R.M., Alcalá-Martínez, E., Sandoval-Arrieta, M.I. and Campos-Navarro, L.A. (2013) In vitro sensitivity to fluconazole through Vitek II systems, of strains of Candida spp. in patients with oropharyngeal candidiasis and HIV/ AIDS. J. AIDS Clin. Res., 4(8): 230.

18. Steubesand, N., Kiehne, K., Brunke, G., Pahl, R., Reiss, K., Herzig, K.H., Schubert, S., Schreiber, S., Fölsch, U.R., Rosenstiel, P. and Arlt, A. (2009) The expression of the $\beta$-defensins HBD-2 and HBD-3 is differentially regulated by NF- $\mathrm{kB}$ and MAPK/AP-1 pathways in an in vitro model of Candida esophagitis. BMC Immunol., 10: 36.

19. Etzioni, A. (2011) Fungal infections: Blame the TH-17 cells. Isr. Med. Assoc. J., 13(9): 561-563.

20. Dejima, T., Shibata, K., Yamada, H., Hara, H., Iwakura, Y., Naito, S. and Yoshikai, Y. (2011) Protective role of naturally occurring interleukin-17A-producing $\gamma \delta \mathrm{T}$ cells in the lung at the early stage of systemic candidiasis in mice. Infect. Immun., 79(11): 4503-4510.

21. Steinmann, J., Bauer, J., Pietschmann, T. and Steinmann, E. (2013) Anti-infective properties of epigallocatechin-3-gallate (EGCG), a component of green tea. Br. J. Pharmacol., 168: 1059-1073.

22. Masatomo, H. and Kazuko, T. (2014) Multiple effects of green tea catechin on the antifungal activity of antimycotics against Candida albicans. J. Antimicrob. Chemother., 53: 225-229.

23. Shibata, N., Kobayashi, H. and Suzuki, S. (2012) Immunochemistry of pathogenic yeast, Candida species, focusing on mannan. Proc. Jpn. Acad., Ser. B. Phys. Biol. Sci., 88(6): 250-265.

24. Navarro-Martínez, M.D., García-Cánovas, F. and Rodríguez-López, J.N. (2006) Tea polyphenol epigallocatechin-3-gallate inhibits ergosterol synthesis by disturbing folic acid metabolism in Candida albicans. J. Antimicrob. Chemother.,57: 1083-1092.

25. Miramón, P., Kasper, L. and Hube, B. (2013) Thriving within the host: Candida spp. interactions with phagocytic cells. Med. Microbiol. Immunol., 202: 183-195. 